\title{
Avaliação Ultra-sonográfica das Variações do Volume Uterino
}

\author{
Ultrasonographic Evaluation of Uterine Volume Variations
}

Francisco Mauad Filho, Augusto Fernando Beduschi, Renata Alberge Giugliano Meschino Fernando Marum Mauad, Mauro da Silva Casanova*, Adilson Cunha Ferreira

\begin{abstract}
RESUMO
Objetivo: verificar a acurácia do ultra-som transabdominal (USTA) em determinar as alterações fisiológicas e patológicas do volume uterino e comparar o volume do útero ao USTA e ultrasom transvaginal (USTV), procurando verificar a relação dos métodos.

Métodos: inicialmente foram revistos, retrospectivamente, 1186 exames ecográficos pélvicos (USTA e USTV), verificando-se as principais doenças e indicações para estes exames. A seguir, foram selecionados 480 USTA sem doenças uterinas e correlacionou-se o volume uterino com a idade e paridade. Finalmente, foi realizado estudo prospectivo comparando-se o volume uterino de 50 mulheres ao USTA e USTV. Para análise estatistica utilizaram-se os testes t de Student e coeficientes de correlação de Spearman e Pearson.

Resultados: correlacionando-se com a paridade, para $P=O(n=99)$ o volume foi de 44,4 $\mathrm{cm}^{3}$; para $P=1(n=72)$ o volume foi $58,5 \mathrm{~cm}^{3}$; para $P=2-3(n=137)$, o volume foi $75,8 \mathrm{~cm}^{3}$; para $P=4-5(n=56), 88 \mathrm{~cm}^{3}$ e para $P=6$ ou mais $(n=26), 105 \mathrm{~cm}^{3}$, mostrando uma correlação positiva entre a paridade e o volume uterino. Com o coeficiente de correlação de Spearman obtiveram-se $r=0,59$ e $p=0,001$; o coeficiente de correlação de Pearson foi $r=0,55$ e o mesmo $p$. Não houve variações significantes do volume uterino ao USTA e USTV.

Conclusões: o útero aumenta com a paridade e sofre alterações do seu volume com a idade, sendo essas modificações detectadas ao USTA. Ambos os métodos (USTA e USTV) são equivalentes na medida do volume uterino; entretanto, a repleção vesical ao USTA permite melhor avaliação do comprimento uterino.
\end{abstract}

PALAVRAS-CHAVE: Útero, volume. Ultra-sonografia transvaginal.

\section{Introdução}

O útero é um dos órgãos mais dinâmicos no corpo humano. O seu volume se modifica assim como sua configuração, sob a influência hormonal da puberdade, na fase de menacme alcança seus diâmetros máximos, atrofiando com a menopausa. Durante a gravidez o útero deve crescer significativamente e modificar-se para proteger, sustentar e finalmente expulsar o feto em desenvolvimento ${ }^{1}$.

Sempre foi motivo de preocupação a determinação precisa do volume uterino, uma vez que

Trabalho realizado na Escola de Ultra-Sonografia e Reciclagem Médica Ribeirão Preto - EURP.

*Bolsista Fapesp

Correspondência:

Francisco Mauad Filho

Rua Casemiro de Abreu n 660 - Vila Seixas

14020-060 - Ribeirão Preto - São Paulo freqüentemente esse órgão é sede de doenças como miomatose, adenomiose e neoplasia trofoblástica gestacional, que podem aumentar muito o seu volume, gerando sintomas compressivos e sangramentos de dificil controle, sendo freqüentemente necessário intervenção cirúrgica de emergência. Além das diversas doenças, o útero sofre mudanças fisiológicas que alteram os seus diâmetros; durante o período reprodutivo, as alterações cíclicas hormonais, a idade e a paridade fazem com que este órgão apresente modificações do seu tamanho ${ }^{2}$.

Entretanto, qual o método a ser utilizado quando necessita-se de uma mensuração uterina confiável? O toque bimanual é pouco preciso e tem limitações quanto ao diagnóstico diferencial da origem do aumento de volume. Com a introdução da ultra-sonografia na década de 70 , esta passou a ser o método de escolha e padrão ideal de imagem para a avaliação do tamanho e das doenças 
uterinas. É um método rápido, preciso e nãoinvasivo, de baixo custo e que não utiliza radiação ionizante ou meios de contraste. Com o advento da sonda vaginal, em meados de 80 , a ultrasonografia transvaginal firmou-se como método de excelência no estudo da pelve feminina ${ }^{1,3}$, assim como para avaliação da gestação no primeiro trimestre, tornando-se exame complementar imprescindivel para a monitorização da gravidez ${ }^{4}$.

Para realização do exame ultra-sonográfico ginecológico, devemos observar se a distenção da bexiga está adequada para a realização do exame. Esta apresenta-se como uma estrutura anecóica com paredes delgadas e lisas que desloca o intestino delgado e o mesentério para fora da pélvis menor e curvando a vagina, a cérvix e o útero posteriormente, para uma posição horizontalizada. Deve-se evitar realizar o exame com a bexiga pouco ou muito distendida.

Pode-se observar no útero os seus contornos, a serosa, a textura miometrial e a espessura endometrial, correlacionando-se o aspecto desta última com a fase do ciclo menstrual. Quanto aos ovários, pode-se obter dados referentes a sua localização, forma, contornos e textura interna.

Com a utilização da sonda vaginal não é necessário a repleção vesical, e por isso a grande maioria das mulheres aceita essa técnica. A sonda vaginal é recoberta por um ou dois preservativos que previamente foram preenchidos com gel. Introduz-se vagarosamente a sonda vaginal e, procurando manter-se contato com a parede vaginal anterior, visualizam-se a uretra e a bexiga quase vazia ${ }^{4}$.

Todavia, ambos os métodos apresentam vantagens e limitações e ainda não se sabe ao certo qual é o mais preciso, mas sabe-se que a correlação entre o exame transabdominal e endovaginal ajuda a esclarecer o diagnóstico de imagens pélvicas complexas e a obtenção de medidas do útero e dos ovários.

Devido à grande importância do assunto, os objetivos desse estudo são verificar o comportamento do volume uterino ao exame ultrasonográfico transabdominal durante a vida reprodutiva da mulher e determinar a relação na medida do volume uterino, comparando-se o ultrasom pélvico transabdominal ao ultra-som transvaginal.

\section{Pacientes e Métodos}

Inicialmente foram avaliados retrospectivamente 1.186 exames ultra-sonográficos pélvicos transabdominais e transvaginais realizados no período de janeiro a agosto de 1999 na Escola de Ultra-Sonografia e Reciclagem Médica Ribeirão Preto (EURP). Destes, foram selecionados 480 exames transabdominais que não mostraram doenças uterinas e procurou-se correlacionar o volume uterino com a idade e, nas mulheres em fase de menacme (19-48), correlacionou-se com a paridade. Posteriormente, realizou-se estudo prospectivo durante o período de janeiro e fevereiro do ano 2000, no qual foram realizados exames em cinqüenta mulheres.

Procedia-se primeiramente ao exame por via transabdominal (transdutor de 3,5 MHz) com aparelho da marca Hitachi 550, com a paciente com a bexiga repleta e realizava-se mensuração uterina nas secções longitudinal (DL), ântero-posterior (DAP) e transversa (DT). Solicitava-se então à paciente que esvaziasse a bexiga e, em posição ginecológica, procedia-se ao exame por via transvaginal (trandutores de 5 a $6,5 \mathrm{MHz}$ ) com aparelho da marca Hitachi 550 e à mensuração uterina nas mesmas três secções, sendo que na longitudinal colo e corpo eram medidos separadamente nos casos em que não era possivel a mensuração dos dois segmentos juntos. O volume uterino foi calculado pela fórmula: DL X DAP X DT $X 0,45$. Nesta etapa também foram consideradas somente as pacientes sem doença uterina.

Este trabalho foi aprovado pela Comissão de Ética em Pesquisa do Hospital das Clínicas da Faculdade de Medicina de Ribeirão Preto da Universidade de São Paulo. Para análise estatística da correlação do volume uterino de acordo com a paridade foram utilizados os coeficientes de correlação de Spearman e Pearson. Para a comparação do volume uterino ao ultra-som transabdominal e transvaginal foi aplicado o teste $t$ de Student.

\section{Resultados}

O total de exames realizados nesse período foi de 1.186. Quanto à procedência das pacientes, a EURP atende basicamente o município de Ribeirão Preto e, em menor número, outras cidades dos Estados de São Paulo e Minas Gerais.

Na Figura 1, correlacionando o volume uterino com a idade, verifica-se que entre 10 e 18 anos $(\mathrm{n}=87)$ o volume uterino médio observado foi de $39 \mathrm{~cm}^{3}$; entre 19 e $48(\mathrm{n}=390)$, o volume foi de $66,8 \mathrm{~cm}^{3}$; entre 49 e $59(\mathrm{n}=48)$, o volume foi de $62,7 \mathrm{~cm}^{3}$, e nas pacientes com idade maior ou igual a 60 anos $(n=21)$ o volume foi $28,5 \mathrm{~cm}^{3}$.

Correlacionando-se com a paridade (p) (Tabela 1) observamos que para $P=0(n=99)$ o volume foi de $44,4 \mathrm{~cm}^{3}$; para $\mathrm{P}=1(\mathrm{n}=72)$, o volume foi 
$58,5 \mathrm{~cm}^{3} ;$ para $\mathrm{P}=2-3(\mathrm{n}=137)$, o volume foi 75,8 $\mathrm{cm}^{3}$; para $\mathrm{P}=4-5(\mathrm{n}=56), 88 \mathrm{~cm}^{3}$ e para $\mathrm{P}=6$ ou mais ( $\mathrm{n}=26), 105 \mathrm{~cm}^{3}$, mostrando uma correlação positiva entre a paridade e o volume uterino. Com o coeficiente de correlação de Spearman obteve-se um $r=0,59$ e $p=0,005$; o coeficiente de correlação de Pearson foi $r=0,55$ para o mesmo $\mathrm{p}$. Calculando-se os valores correspondentes ao $10^{\circ}$, $50^{\circ}$ e $90^{\circ}$ percentis, respectivamente (Tabela 1 ), obtiveram-se para $\mathrm{P}=0$ os valores $(28 ; 41 ; 65) ; \mathrm{P}=$ 1 (34; 56; 92,5); $\mathrm{P}=2$ e 3 (43; 70; 115); $\mathrm{P}=4$ e 5 (49; $82,5 ; 137) ; \mathrm{P}=6$ ou mais $(61 ; 89 ; 166,5)$.

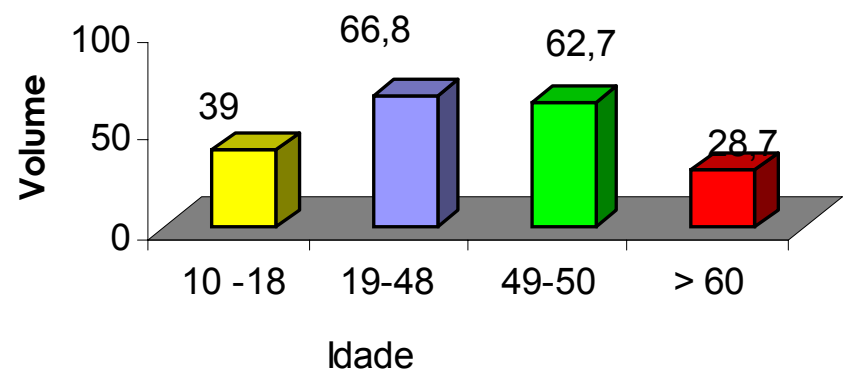

Figura 1 - Correlação do volume uterino $\left(\mathrm{cm}^{3}\right)$ com a idade.

Tabela 1 - Valores do volume uterino $\mathrm{em} \mathrm{cm}^{3}$, no $10^{\circ}, 50^{\circ}$ e $90^{\circ}$ percentis, de acordo com a paridade na fase de menacme.

\begin{tabular}{lllcrc}
\hline Percentil & $\mathbf{P}_{\mathbf{0}}$ & $\mathbf{P}_{\mathbf{1}}$ & $\mathbf{P}_{\mathbf{2}}$ & $\mathbf{P}_{3}$ & $\mathbf{P}_{4}$ \\
\hline 10 & 28 & 34 & 43 & 49 & 61 \\
50 & 41 & 56 & 70 & 82 & 89 \\
90 & 65 & 92 & 115 & 137 & 166 \\
Volume $\left(\mathrm{cm}^{3}\right)$ & 44,4 & 58,5 & 75,8 & 88 & 105 \\
\hline
\end{tabular}

$P_{0}=$ nunca pariu; $P_{1}=$ pariu uma vez; $P_{2}=$ pariu duas ou três vezes; $P_{3}=$ pariu quatro ou cinco vezes; $P_{4}=$ pariu seis ou mais vezes

$\mathrm{Na}$ análise comparativa da mensuração uterina efetuada com o USTA e com o USTV observam-se os valores médios com seus respectivos desvios-padrão (DP) mostrados na Figura 2.

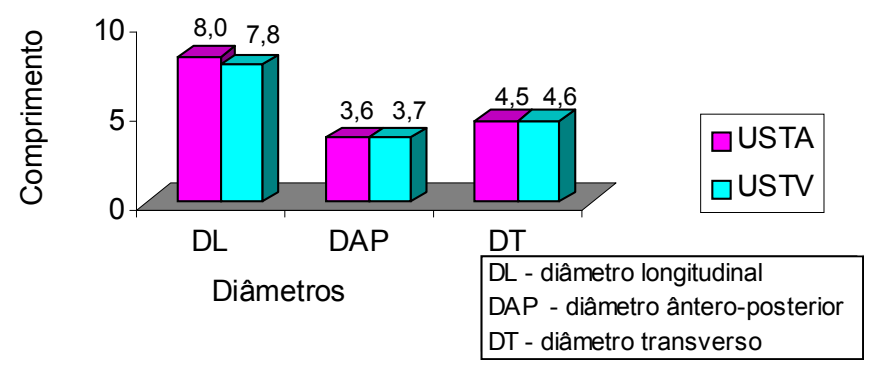

Figura 2 - Análise comparativa dos comprimentos (cm) dos diâmetros uterinos (DL, DAP, DT) medidos pela ultra-sonografia transvaginal (USTA) e transvaginal (USTV).
$\mathrm{Na}$ análise comparativa do volume uterino ao USTA e USTV, não houve variações ou diferenças significativas, obtendo-se $62,5 \mathrm{~cm}^{3}$ quando realizado o USTA e $69,8 \mathrm{~cm}^{3}$ ao USTV (Tabela 2). Somente o diâmetro longitudinal (DL) apresentou diferença estatística significante, sendo maior ao USTA.

Tabela 2 - Valores do volume uterino à USTA (ultra-sonografia transvaginal) e USTV (ultra-sonografia transvaginal).

\begin{tabular}{|c|c|c|c|c|c|}
\hline & \multicolumn{2}{|c|}{ USTA } & \multicolumn{2}{|c|}{ USTV } & \multirow[t]{2}{*}{$P$} \\
\hline & VM & DP & VM & $\mathrm{DP}$ & \\
\hline $\mathrm{DL}$ & 8,1 & 0,2 & 7,8 & 0,2 & 0,02 \\
\hline DAP & 56 & 0,4 & 3,6 & 0,1 & 0,45 \\
\hline DT & 4,5 & 0,2 & 4,6 & 0,1 & 0,33 \\
\hline Volume & \multicolumn{2}{|c|}{62,45} & \multicolumn{2}{|c|}{69,74} & 0,07 \\
\hline
\end{tabular}

$\mathrm{VM}=$ valor médio; $\mathrm{DP}=$ desvio-padrão; $\mathrm{P}=$ Pearson; $\mathrm{DL}=$ diâmetro longitudinal; $\mathrm{DAP}=$ diâmetro ântero-posterior; DT = diâmetro transverso

\section{Discussão}

O presente estudo evidenciou modificações do volume uterino com a idade, sendo que o seu crescimento inicia-se na puberdade, os maiores volumes foram observados entre 19 e 48 anos e a partir dos 49 começa a involução, com atrofia após os 60 anos. Estes achados são concordantes com os estudos de Piiroinen e Kaihola ${ }^{2}$, Gross et al. ${ }^{4}$, Orsini et $a .^{5}$, que relatam não haver mudanças significativas do tamanho do útero até o início da puberdade, época a partir da qual ele cresce de forma rápida e homogênea. Para a maioria dos autores, o crescimento uterino começa a partir dos 10 anos $^{5-7}$.

Essas mudanças fisiológicas do tamanho uterino de acordo com a idade refletem-se no colo e corpo do órgão. O colo corresponde a $50 \%$ do comprimento total do útero em um grupo de crianças de 7 a 12 anos de idade ${ }^{8}$. Já no grupo de jovens com idade entre 13 e 17 anos o colo corresponde a $40 \%$ do comprimento total do órgão. Influências hormonais iniciadas na puberdade dão ao útero da mulher adulta a sua forma característica, correspondendo o corpo uterino a dois terços da medida longitudinal total ${ }^{8}$. Portanto, o útero tem um pequeno volume pré-puberal, um crescimento a partir da puberdade, valores máximos na fase de menacme e involução na menopausa. Esta involução é proporcional aos anos de pós-menopausa ${ }^{9}$.

O formato piriforme é típico do período pré- 
puberal, sendo que a relação corpo/ cérvice é de 1:4 e pode ser claramente demonstrada pela ultrasonografia. A partir dos sete anos de idade o útero cresce, com aumento de todos os seus diâmetros e volumes, porém seu crescimento é mais pronunciado no corpo do que na região cervical, e a relação corpo/cérvice, que na puberdade é de $1: 1$, na adulta chega a ser de $2: 1$. A cada gestação ocorre aumento considerável das dimensões uterinas, as quais nunca retornam aos valores iniciais. Portanto, o útero tende a ser de maior volume em multíparas que em nulíparas ${ }^{1}$. Com a redução dos niveis de estrogênio e progesterona na pós-menopausa ocorre diminuição das dimensões uterinas e inversão da relação corpo/cérvice que passa a ser 1:1 ou 1:2. A redução das dimensões uterinas ocorre com maior rapidez nos primeiros 10 anos da menopausa, sendo mais lenta nos anos subseqüentes ${ }^{3}$.

Analisando-se as variações do volume uterino de acordo com a paridade, ficou evidente neste estudo uma correlação positiva com o aumento progressivo deste órgão. Platt et al. ${ }^{9}$ relataram um peso de $60 \mathrm{~g}$ em nulíparas, de $109 \mathrm{~g}$ em primiparas e de $108 \mathrm{~g}$ em multiparas. Mostrou-se também correlação positiva com a idade e parida$\mathrm{de}^{10,11}$.

Está claro que o útero é órgão passivel de modificações fisiológicas do seu volume, entretanto, uma estimativa do volume uterino somente pelo toque bimanual pode ser dificil em pacientes obesas e com dor pélvica. A ultra-sonografia tem revolucionado as condutas na prática ginecológica, permitindo uma perfeita visualização da pelvis e uma determinação precisa do volume uterino pela medida dos três diâmetros, longitudinal, ânteroposterior e transverso, além de diagnosticar as principais causas de aumento do volume desse órgão e de ser técnica não-invasiva, de baixo custo e com boa aceitação pelas pacientes ${ }^{12}$.

Contudo, a ultra-sonografia transabdominal também apresenta limitações. O fundo uterino normal de um útero retrovertido pode ser confundido com mioma subseroso posterior ${ }^{13}$. Para uma mensuração segura do útero é necessário uma boa repleção vesical, pois com a bexiga vazia não é possível a visualização do fundo uterino, mas a bexiga muito cheia pode provocar uma curvatura inadequada da cérvice e do útero, provocando distorções anatômicas que devem ser evitadas ${ }^{14}$. Em pacientes com repleção vesical deforma-se o ângulo de 90 a 130 graus formado entre o corpo e o colo uterino, situação esta denominada de anteversão. O corpo do útero torna-se mais posteriorizado, aumentando o ângulo entre a vagina e colo além dos 90 a 130 graus habituais, o que impede o estudo desta relação anatômica pela ultra-sonografia suprapúbica ${ }^{15}$. A ultra-sonografia transvaginal apresenta algumas vantagens em relação à ultra-sonografia transabdominal, pois utilizando-se transdutores de alta freqüência a resolução da imagem aumenta, permitindo melhor visualização e com mais detalhes da morfologia uterina. Além de evitar zonas cegas como gases e ossos, é bem aceito pela maioria das mulheres, pois não há o desconforto e se evitam as deformidades anatômicas provocadas pela repleção vesical. Como desvantagem esse método sofre limitações nas grandes tumorações que ultrapassam os limites da pelve, pela maior distância de insonação ${ }^{14}$.

Para uma medida exata do volume uterino é necessária uma boa visualização do colo, o que é permitido ao USTV pela proximidade do transdutor com a cérvice, sendo identificadas as alterações fisiológicas, assim como as doenças benignas e a extensão dos processos malignos ${ }^{16}$. O desconforto para a paciente no exame transvaginal é mínimo, porque a repleção vesical não é necessária.

$\mathrm{Na}$ tentativa de se identificar qual o melhor método para uma avaliação precisa do volume uterino, comparou-se nesse estudo o volume de 50 úteros medidos ao exame ultra-sonográfico transabdominal e transvaginal, verificando-se uma variação estatística significante no diâmetro longitudinal, que foi maior ao USTA. Este fato talvez possa ser explicado pelo aumento do ângulo entre o colo e a vagina com a repleção vesical ${ }^{15}$. $\mathrm{Na}$ análise do volume uterino não houve diferença significativa, concordando com Nazário et al. ${ }^{17}$.

No estudo das anomalias uterinas, pesquisas recentes comparando a USTV e a ressonância magnética (RM) têm indicado que a USTV deve ser o método primário de estudo e recomenda-se a RM para os casos especiais ${ }^{18,19}$.

Na década de 1990, com o auxílio do Doppler colorido, da ultra-sonografia tridimensional e pela possibilidade de complementar o exame pela histerossonografia, RM e tomografia computadorizada houve novos subsídios para avaliação do útero e da pelve feminina, e os estudos devem ser cada vez mais promissores, apresentando avaliações cada vez mais precisas ${ }^{1,12}$.

Do exposto, o trabalho contribui aos estudos morfológicos do volume uterino e suas variações fisiológicas, reforçando o papel da importância da ultra-sonografia na propedêutica ginecológica, por ser método de baixo custo e pouco desconforto, em comparação aos outros métodos existentes. 


\section{SUMMARY}

Purpose: to determine the accuracy of transabdominal ultrasound (TAUS) in revealing physiological and pathological changes in uterine volume, and to compare uterine volume detected by TAUS and by transvaginal ultrasound (TVUS) in an attempt to determine the relationship between the two methods.

Methods: a total of 1,186 pelvic echographic examinations (TAUS and TVUS) were first reviewed retrospectively in terms of the major diseases and indications for the examinations. A total of 480 TAUS examinations without uterine disease were then selected and uterine volume was correlated with age and parity. Finally, a retrospective study was conducted to compare the uterine volume of 50 women obtained by TAUS and TVUS. Data were analyzed statistically by Student's ttest and Spearman and Pearson correlation.

Results: correlating with parity, volume was $44.4 \mathrm{~cm}^{3}$ for $P=$ $0(n=99), 58.5 \mathrm{~cm}^{3}$ for $P=1(n=72), 75.8 \mathrm{~cm}^{3}$ for $P=2-3(n$ $=137), 88 \mathrm{~cm}^{3}$ for $P=4-5(n=56)$, and $105 \mathrm{~cm}^{3}$ for $P=6$ or more $(n=26)$, showing a positive correlation between parity and uterine volume. Calculation of Spearman's correlation coefficient yielded $r=0.59$ and $p=0.001$ and calculation of Pearson's correlation coefficient yielded $r=0.55$ and the same $p$ value. There were no significant differences between the uterine volumes detected by TAUS and TVUS.

Conclusions: the volume increases with parity and changes with age, and these modifications are detected by TAUS. The two methods (TAUS and TVUS) are equivalent in determining the uterine volume; however, when TAUS is used, bladder filling allows a better evaluation of the uterine length.

KEY WORDS: Uterine volume. Ultrasonography, transvaginal.

\section{Referências}

1. Hall DA, Yoder IC. Avaliação ultra-sonográfica do útero. In: Callen PW, editor. Ultra-sonografia em Obstetrícia e Ginecologia. $3^{a}$ ed. São Paulo: Guanabara Koogan; 1994. p.567-94.

2. Piiroinen O, Kaihola HL. Uterine size measured by ultrasound during the menstrual cycle. Acta Obstet Gynecol Scand 1975; 54:247-50.

3. Moura APC, Pastore AR, Cerri GG. Miométrio. In: Pastore AR, Cerri GG, editores. Ultra-sonografia: Obstetrícia, Ginecologia. $1^{a}$ ed. São Paulo: Sarvier; 1997. p.541-54.

4. Gross R, Mauad Filho F, Ayres CE, Baracchini JAA, Mangieri Sobrinho F. Medidas do volume do útero e ovários obtidas através da ultra-sonografia. In: Mauad Filho F, editor. Manual e Coletânea de Tabelas em Ultra-sonografia. $1^{\text {a }}$ ed. Ribeirão Preto: Scala; 1997. p.65-67.
5. Orsini LF, Salardi S, Pilu G, Bovicelli L, Cacciari E. Pelvic organs in premenarcheal girls: real time ultrasonography. Radiology 1984; 153:113-6.

6. Hata K, Nishigaki A, Makihara K, Takamiya O, Hata T, Kitao M. Ultrasonic evaluation of the normal uterus in the neonate. J Perinat Med 1989; $17: 313-7$.

7. Cacciatore B, Apter D, Alfthan H, Stenman UH. Ultrasonic characteristics of the uterus and ovaries in relation to pubertal development and serum LH, FSH and estradiol concentrations. Adolesc Pediatr Gynecol 1991; 4:15-20.

8. Ivarsson SA, Nilsson KO, Persson PH. Ultrasonography of the pelvic organs in the prepubertal and postpubertal girls. Arch Dis Child 1993; 58:352-4.

9. Platt JF, Bree RL, Davidson D. Ultrasound of the normal nongravid uterus: correlation with gross and histopathology. J Clin Ultrasound 1990; 18:15-9.

10.Langlois PL. Size of normal uterus. J Reprod Med 1970; 4:220-8.

11.Haber HP, Mayer EL. Ultrasound evaluation of uterine and ovarian size from birth to puberty. Pediatr Radiol 1994; 24:11-3.

12.Pastore AR, Cerri GG. Dopplervelocimetria em ginecologia. In: Pastore AR, Cerri GG, editores. Ultra-sonografia: Obstetrícia, Ginecologia. $1^{\mathrm{a}}$ ed. São Paulo: Sarvier; 1997; p.639-65.

13.Vilaro MM, Rifkin MD, Pennell RG, et al. Endovaginal ultrasound. A technique for evaluation of nonfolicular pelvic masses. J Ultrasound Med 1987; 6:697-701.

14. Mauad Filho F. Manual e coletânea de tabelas em ultra-sonografia. $1^{\text {a }}$ ed. Ribeirão Preto: Scala; 1997. p.72-82.

15. Carvalho IE, Cerri GG. Anatomia ultra-sonográfica da pelve feminina. In: Pastore AR, Cerri GG, editores. Ultra-sonografia: Obstetricia, Ginecologia. $1^{\mathrm{a}}$ ed. São Paulo: Sarvier; 1997. p.477-97.

16. Cartro FS, Pastore RA. Colo uterino. In: Pastore AR, Cerri GG, editores. Ultra-sonografia: Obstetrícia, Ginecologia. $1^{\mathrm{a}}$ ed. São Paulo: Sarvier; 1997. p.527-39.

17. Nazário ACP, Nicolau SM, Nishimura CM. Comparação entre a ecografia pélvica endovaginal e a transabdominal na mensuração do útero e dos ovários. Rev Paul Med 1991; 109:51-4.

18.Randolph JF Jr, Ying YK, Maier DB, Schmidt CL, Riddick DH, Randolph JR Jr. Comparison of realtime ultrasonography, hysterosalpingography, and laparoscopy/hysteroscopy in the evaluation of uterine abnormalities and tubal patency. Fertil Steril 1986; 46:828-32.

19.Pellerito JS, McCarthy SM, Doyle MB, Glickman MG, Decherney AH. Diagnosis of uterine anomalies: relative accuracy of MR imaging, endovaginal sonography, and hysterosalpingography. Radiology 1992; 183:795-800. 